\title{
An Enhanced Electroporator Design for Pulse Generation
}

\author{
Sadasivam Pachamuthu \\ Electrical and Electronics Engineering \\ B. S. Abdur Rahman Crescent Institute of Science and \\ Technology \\ Vandalur, Chennai, India \\ psvijay92@gmail.com
}

Kavitha Sankaranarayanan

AU - KBC Research Centre MIT

Campus of Anna University

Chennai, India

kavitham@yahoo.com

\author{
D. Najumnissa \\ Electronics and Instrumentation Engineering \\ B. S. Abdur Rahman Crescent Institute of Science and \\ Technology \\ Vandalur, Chennai, India \\ najumnissa.d@crescent.education \\ Raja Prabu Ramachandran \\ Gandhi Institute of Technology and Management \\ Deemed to be University \\ Vishakapatnam, Andhra Pradesh, India \\ director_ar@gitam.edu
}

\begin{abstract}
An electroporator is an instrument used for delivering electrical pulses to a tumor. In this work, an electroporator consisting of three main system blocks, namely High Voltage (HV) source, nanosecond (ns) switching, and pulse generation, was designed, developed, and evaluated to generate high voltage ns pulses to treat tumors. The high-voltage source block was used to convert the $9.6 \mathrm{~V}$ DC from the battery to a variable $\mathrm{HV}$ output and store this charge for later use. The ns switching block contained a MOSFET-based low-side switch which applies short ns pulses to the load. The pulse generation block generates short ns pulses and supplies the adequate current to turn on the MOSFET at a quicker rate aiding the application of these pulses to the load. This process was simulated using PSpice software and the results are presented.
\end{abstract}

Keywords-electroporator; electrochemotherapy; boost converter microcontroller;

\section{INTRODUCTION}

Cancer is the second most lethal disease, as it is responsible for almost the $13 \%$ of all human deaths. The extra economic burden related to cancer complicates the administration of presently adopted remedy strategies [1]. Reversible electroporation is the process where a short electric field on a cell retains the membrane's ability to recover at a quicker rate, while the cell remains viable. On the other hand, irreversible electroporation is the process where longer and high-intensity pulses are applied breaking down the cell membrane irreversibly [2]. Reversible electroporation can be further improved or optimized [3]. It is also feasible to electroporate using very brief and very strong electric pulses [4]. In this study, a new electroporator to produce nanosecond (ns) high voltage pulses for treating abnormalities of skin cells is designed, developed, and evaluated. The electroporator instrument consists of a pulse generator and an electrode for delivering the pulse to the tumor's location. The pulse generator produces an electric pulse of predetermined magnitude, width, and frequency. The basic principles used in almost all pulse generators are the capacitor charging and discharging mechanisms. Three system blocks make up the miniature high voltage ns pulse: The High-Voltage (HV) source block converts the battery's $9.6 \mathrm{~V}$ DC into a variable high voltage output, and this charge is stored to use when creating the pulse. The ns switching block is a low-side switch with a MOSFET that sends brief ns pulses to the load. The pulse generation block generates the ns pulses and provides sufficient current to turn the MOSFET on quickly enough to deliver them to the load.

\section{MATERIALS AND METHODS}

The following materials were used:

- Pulse generator: A logic-based PIC controller was used for creating the ns pulses using a program written in mikroC, converted into hex code, and loaded to the microcontroller.

- Programmable Integrated Circuit (PIC) Controller: The PIC controller featured program memory, SRAM, EEPROM, analog comparators, and Pulse With Modulation (PWM). All these characteristics make it appropriate for more advanced analog to digital applications. Using a PIC as a master controller to generate the PWM and interface with the LCD and the keypad reduced the required components and made the electroporator more compact.

- HV source block: 0-12V DC was converted to 0-1000V DC using the HV module. The $230 \mathrm{~V}$ AC supply was connected to an adjustable voltage regulator, which fed the HV module. A potentiometer was used to set the regulator voltage around 9VDC and obtain a $1000 \mathrm{~V}$ output. Two diodes were utilized to safeguard the HV module's output from transient spikes and damage. As the HV module was 
rated at $10 \mathrm{~W}$, a $150 \mathrm{k}$ resistor was used to limit the current to $6.6 \mathrm{~mA}$ and not exceed the module's rated output power. When the module was pulsed, it charged a $1 \mathrm{uF}$ capacitor which gave energy to the load.

- Ns switch block: The source of the MOSFET was connected to the ground, and the drain was connected to the load's negative side. A fast switching diode was placed across it to reduce ringing on the load. A $150 \Omega$ resistor was connected in parallel with the load to aid in measurements using an oscilloscope.

- LCD: An LCD 4×16 display was used (Figure 1).

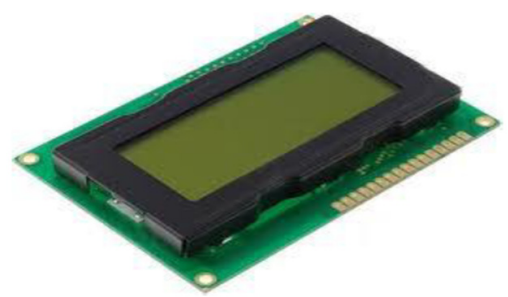

Fig. 1. $4 \times 16 \mathrm{LCD}$.

- Keypad: The keypad interfaced with the PIC for obtaining input from the user. A $4 \times 4$ keypad was used, having the 0-9 digits and four keys for other operations. The keypad was used to select the parameters and protocols according to the program in the PIC microcontroller.

\section{PROPOSED ELECTROPORATOR DESIGN}

In this work, a programmable electroporator using the PIC16F877A microcontroller was designed and developed [5]. Figure 2 shows the schematic arrangement of the programmable electroporator. The designed electroporator consists of a microcontroller, a power circuit as an electroporator, and an LCD. The electric supply is given to the electroporator through a boost converter. A logic-based PIC controller is used for programming the pulses using embedded C. The program was written in MikroC Integrated Development Environment (IDE), converted into hex code, and loaded to the microcontroller. The pulses were generated at the output PORTB pin. The microcontroller can store up to 16 programs for different applications, and the user can select which fits its needs. Figure 3 shows the boost converter's control diagram used for generating the high voltage supply for electroporation. The AC input is converted to a high voltage AC supply using the HV transformer and rectifier [6-8]. There are many ways to achieve a $1000 \mathrm{~V}$ potential. One could be to transform $120 \mathrm{VAC}$ and then rectify it to $1000 \mathrm{~V}$. This was not chosen because of the large size and weight of the needed transformer and rectifier. This study's motive was to keep the pulser small and portable using a high voltage DC-DC converter module (0-12VDC to $0-1500 \mathrm{VDC})$. The battery voltage was applied to the variable voltage regulator and the high voltage module. A potentiometer was used to adjust the regulator voltage up to $8 \mathrm{VDC}$ to obtain an output voltage of around $1000 \mathrm{~V}$. Figure 4 shows the booster voltage display and Table I presents a comparison between an existing and the designed electroporator.

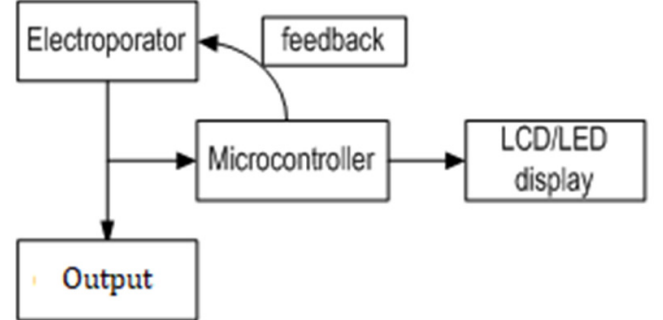

Fig. 2. Schematic diagram of the programmable electroporator.

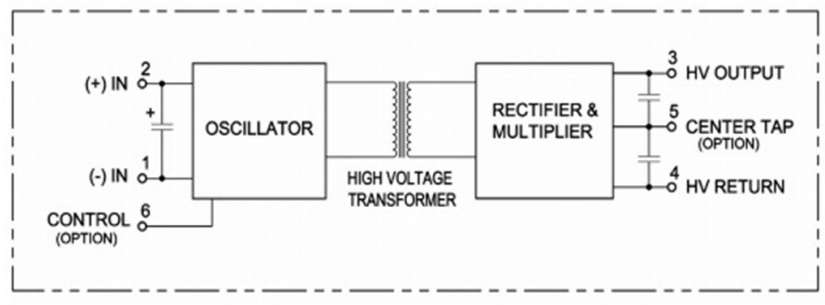

Fig. 3. Boost converter control diagram.

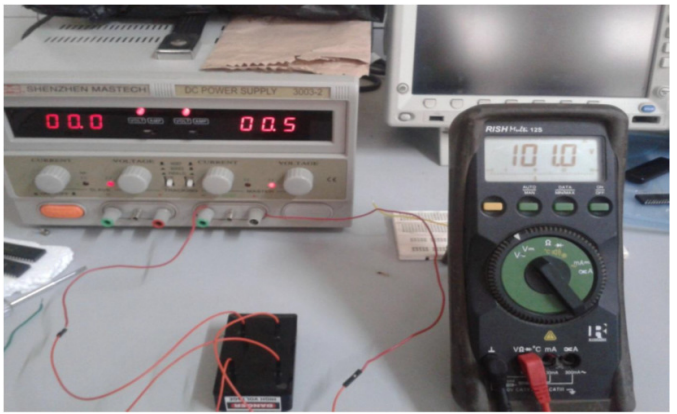

Fig. 4. Boosted voltage displayed in voltmeter.

TABLE I. COMPARISON BETWEEN EXISTING AND DEVELOPED MODELS OF ELECTROPORATOR DESIGN

\begin{tabular}{|c|c|}
\hline Existing model ECM 830 [9] & Developed model \\
\hline $\begin{array}{c}\text { Has more components for pulse } \\
\text { generation. }\end{array}$ & Uses a PIC controller \\
\hline $\begin{array}{c}\text { Uses an RC network and flip flops } \\
\text { before sending the pulses to the IC } \\
\text { driver }\end{array}$ & $\begin{array}{c}\text { Uses an isolator between the } \\
\text { pulse and the driver }\end{array}$ \\
\hline $\begin{array}{c}\text { Cannot use display and input device } \\
\text { without having a separate processor } \\
\text { which further increases its cost. }\end{array}$ & $\begin{array}{c}\text { Already uses a controller so } \\
\text { LCD and keypad can be easily } \\
\text { attached. }\end{array}$ \\
\hline Must be changed manually. & $\begin{array}{c}\text { Logically controlled so multiple } \\
\text { programs can be executed in a } \\
\text { single device. }\end{array}$ \\
\hline
\end{tabular}

\section{A. Switch Block Description}

Pulses having a duration as low as 20ns were required in the design specifications; therefore an extremely fast RF MOSFET was used, as shown in Figure 5. A 5ns rise time $1200 \mathrm{~V}$ MOSFET was chosen as a switch. A high current driver was used to switch the MOSFET. The $1960 \mathrm{pF}$ capacitance of the MOSFET was charged through an initial high current draw using this driver. The 4 ns rise time MOSFET driver was used 
to make the driver act very fast. The battery was used to charge the MOSFET driver with no specific regulations. The Complementary Metal-Oxide-Semiconductor (CMOS) and the Transistor-Transistor Logic (TTL) were the inputs of the MOSFET driver, which were connected to the pulse generation circuit output.

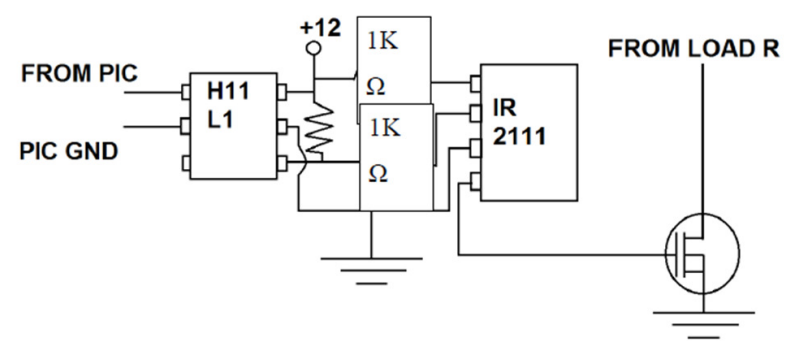

Fig. 5. ns switch design.

Two CMOS digital Integrated Circuits (ICs) were used to construct a ns pulse generator circuit. Those two ICs were retriggerable monostable multivibrator and D flip flop. The pulses were produced by the re-triggerable monostable multivibrator, determined by the value of one external capacitor and resistor. The multivibrator was a dual IC, so using one multivibrator two pulses can be generated, and the pulses can be shifted by increasing the external resistor of the multivibrator. A variable resistance was connected in series with the external resistor for allowing pulse duration adjustments. These pulses were then used to clock and preset the D flip flop. Separating the pulses by 20 ns allows the D flip flop to be clocked high and then preset low 20ns later. The retriggerable monostable multivibrator needs a clock signal to repeat the pulses. A 555 timer was used to create a CMOS clock of $1.5 \mathrm{kHz}$. The 555 timer was also built with potentiometers to allow adjustment of frequency and duty cycle. All three ICs were powered using a simple 5VDC regulator.

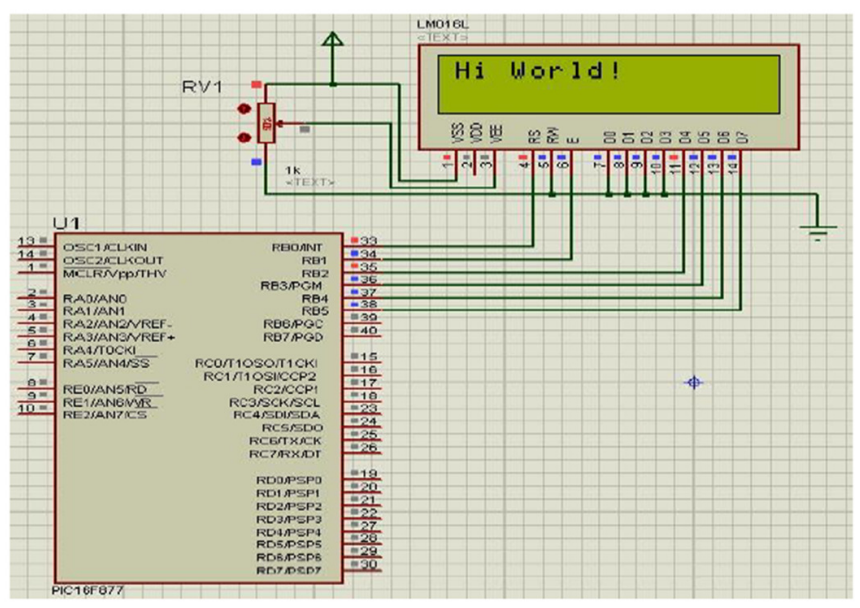

Fig. 6. Interfacing PIC with LCD.

\section{B. Interfacing PIC with $L C D$}

The PIC was interfaced with the LCD, as shown in Figure 6 , for displaying the output after the pulse. Simple code was used for the interface, as the interfacing of an LCD and a keypad to a PIC is simple. The number of delivered pulses was displayed in the LCD through software.

\section{Hardware Circuit Testing and Modeling of Electroporator}

All hardware components (pulse generator, PIC controller, HV source block, ns switch block, LCD, and keypad) were connected and tested in the laboratory, as shown in Figure 7, and the results were evaluated.

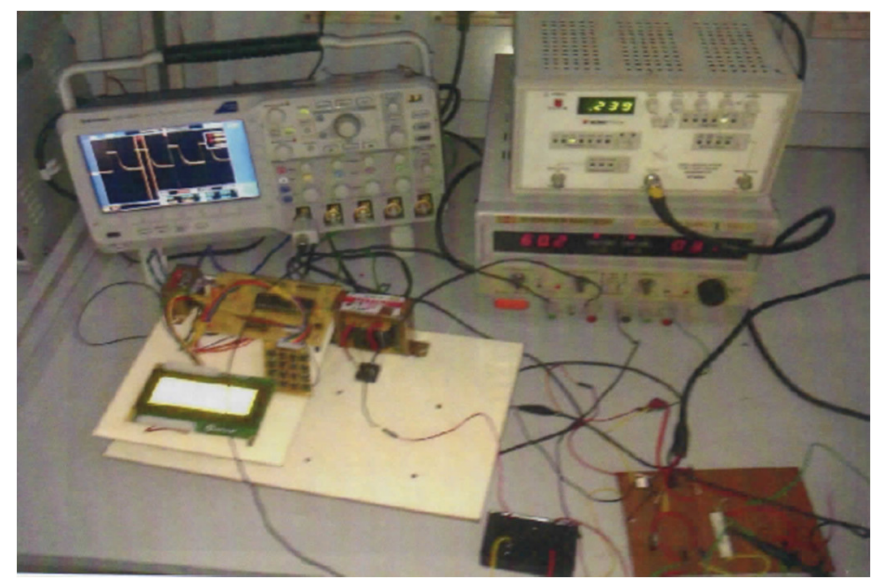

Fig. 7. Testing and modeling of the electroporator.

D. Steps for the Electroporator Using Various Applications

- Display the parameters to control the output of the electroporator.

- Choose a parameter, give an in-range value, and press OK.

- Pulses are applied through the electrodes.

- The number of pulses applied will be displayed.

- Similarly, repeat for all the parameters and press OK.

\section{RESULTS AND DISCUSSION}

\section{A. Output Waveforms}

The results depicted in Figures 8-12 show the effect of electric pulses of $50 \mathrm{mV}$ magnitude, in both positive and negative, in cancer cell HEP using the patch-clamp technique and obtained a single ion channel output which proves the electroporation effect. This technique provides knowledge on the permeability and the changes that occur while electric pulses are delivered to the cell membrane [10]. The electroporator consists of electrodes delivering high voltage pulses to the affected site, and other electronic components such as power converters to convert the low voltage to high $[11,12]$. 


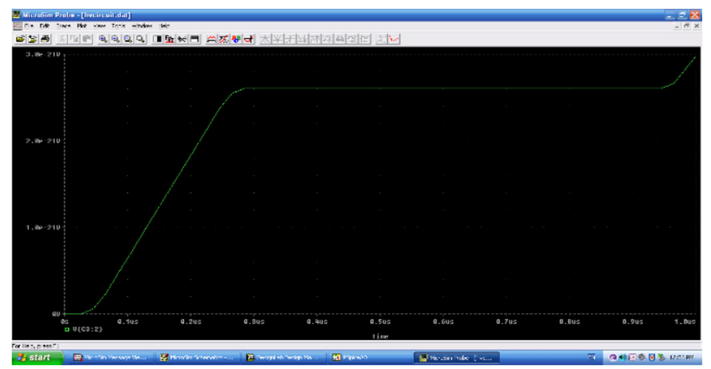

Fig. 8. Transient Voltage graph of the HV block.

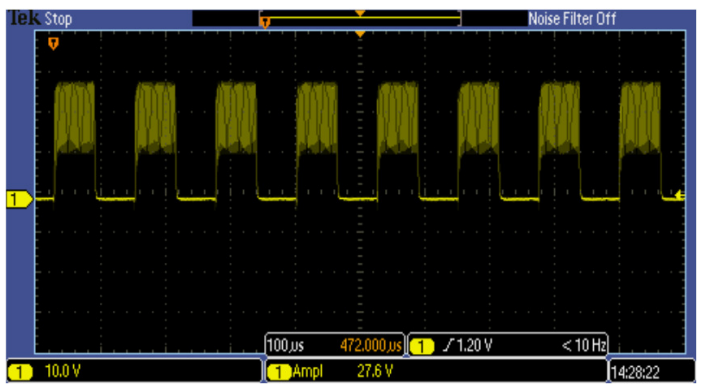

Fig. 9. The obtained output without the capacitor to reduce noise.

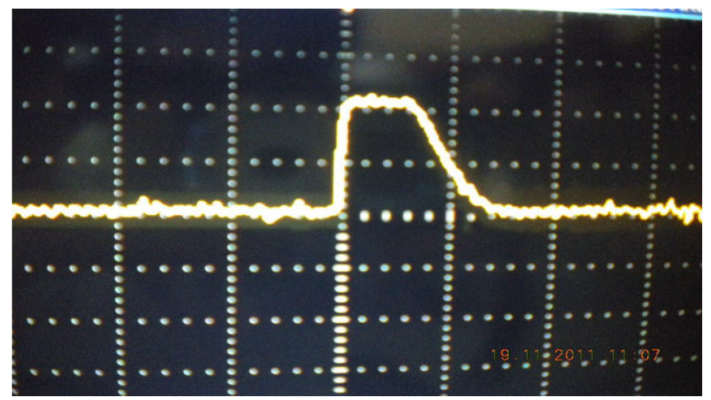

Fig. 10. The output of BTX ECM 830

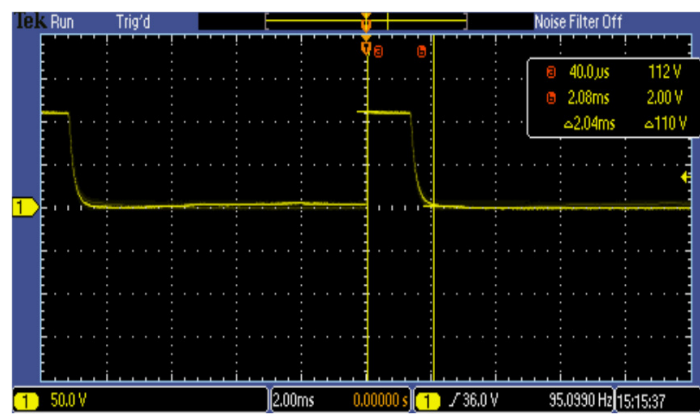

Fig. 11. Single pulse output of RAPKVISS

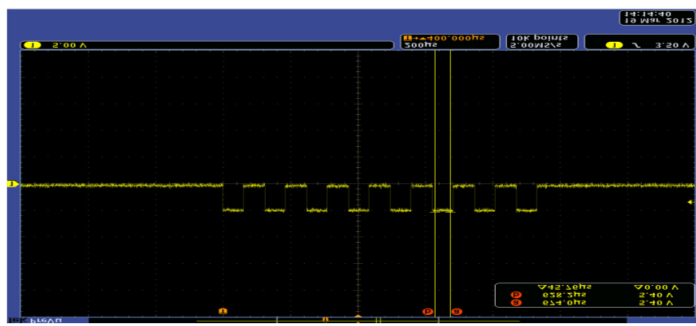

Fig. 12. The output of RAPKVISS for skin cancer.

\section{CONCLUSION}

Electrochemotherapy is an effective technique that uses high voltage electrical pulses to build up the drug transport across the plasma cell membranes. Moreover, it controls the drug proliferation rate by cancer cell apoptosis, as their morphological properties are identical to that of a human stem cell. In this paper, an electroporator was designed and developed to enhance the drug delivery in electrochemotherapy, using a PIC microcontroller and a high voltage boost converter. The proposed electroporator had an LCD to display the parameters of the pulses delivered to the cancer cells. The effect of electric pulses was analyzed and a single ion channel output that proves the electroporation effect was obtained. The main advantages of the proposed electroporator are its low cost and its controllability, as multiple programs can be executed in a single electroporator. Thus, the proposed electroporator was proved to be more advantageous over existing models and had additional features, like displaying the applied pulses with their parameters and a $4 \times 4$ keypad for data input to control its output. Furthermore, it produced a more squarely output than any other electroporator existing to date. Thus, the proposed design can be a costeffective and efficient pulse generator for electroporation in many applications such as gene transplantation, immunotherapy, and food processing for protection against microbes.

\section{REFERENCES}

[1] J. C. Weaver, "Electroporation of cells and tissues," IEEE Transactions on Plasma Science, vol. 28, no. 1, pp. 24-33, Feb. 2000, https://doi.org/ $10.1109 / 27.842820$.

[2] L. M. Mir, O. Tounekti, and S. Orlowski, "Bleomycin: Revival of an old drug," General Pharmacology: The Vascular System, vol. 27, no. 5, pp. 745-748, Jul. 1996, https://doi.org/10.1016/0306-3623(95)02101-9.

[3] L. M. Mir, "Therapeutic perspectives of in vivo cell electropermeabilization," Bioelectrochemistry, vol. 53, no. 1, pp. 1-10, Jan. 2001, https://doi.org/10.1016/S0302-4598(00)00112-4.

[4] T. B. Napotnik, M. Reberšek, T. Kotnik, E. Lebrasseur, G. Cabodevila, and D. Miklavčič, "Electropermeabilization of endocytotic vesicles in B16 F1 mouse melanoma cells," Medical \& Biological Engineering \& Computing, vol. 48, no. 5, pp. 407-413, May 2010, https://doi.org/ 10.1007/s1 1517-010-0599-9.

[5] A. Gothelf, L. M. Mir, and J. Gehl, "Electrochemotherapy: results of cancer treatment using enhanced delivery of bleomycin by electroporation," Cancer Treatment Reviews, vol. 29, no. 5, pp. 371387, Oct. 2003, https://doi.org/10.1016/S0305-7372(03)00073-2.

[6] C. T. S. Ching, T. P. Sun, W. T. Huang, S. H. Huang, C. S. Hsiao, and K. M. Chang, "A circuit design of a low-cost, portable and programmable electroporation device for biomedical applications," Sensors and Actuators B: Chemical, vol. 166-167, pp. 292-300, May 2012, https://doi.org/10.1016/j.snb.2012.02.065.

[7] A. Al-Ateeq and A. J. Alateeq, "Soft-Charging Effects on a High Gain DC-to-DC Step-up Converter with PSC Voltage Multipliers," Engineering, Technology \& Applied Science Research, vol. 10, no. 5, pp. 6323-6329, Oct. 2020, https://doi.org/10.48084/etasr.3773.

[8] M. Taherian, M. Allahbakhshi, E. Farjah, and H. Givi, "A Modular Topology of Marx Generator Using Buck-Boost Converter," IEEE Transactions on Plasma Science, vol. 47, no. 1, pp. 549-558, Jan. 2019, https://doi.org/10.1109/TPS.2018.2876474.

[9] BTX Harvard Apparatus, Holliston MA, USA, ECM 830 Electroporation System User's Manual, Accessed: Aug. 29, 2021. [Online]. Available: https://www.btxonline.com/media/wysiwyg/ tab content/ECM 830.pdf 
[10] J. Cutrera, M. Torrero, K. Shiomitsu, N. Mauldin, and S. Li, "Intratumoral Bleomycin and IL-12 Electrochemogenetherapy for Treating Head and Neck Tumors in Dogs," in Electroporation Protocols: Preclinical and Clinical Gene Medicine, S. Li, Ed. Totowa, NJ, USA: Humana Press, 2008, pp. 319-325.

[11] P. Vu, D. T. Anh, and H. D. Chinh, "A Novel Modeling and Control Design of the Current-Fed Dual Active Bridge Converter under DPDPS Modulation," Engineering, Technology \& Applied Science Research, vol. 11, no. 2, pp. 7054-7059, Apr. 2021, https://doi.org/10.48084/ etasr.4067.

[12] B. Zhao, Q. Song, W. Liu, and Y. Sun, "Overview of Dual-ActiveBridge Isolated Bidirectional DC-DC Converter for High-FrequencyLink Power-Conversion System," IEEE Transactions on Power Electronics, vol. 29, no. 8, pp. 4091-4106, Aug. 2014, https://doi.org/ 10.1109/TPEL.2013.2289913. 